\section{A Computational Analysis of Squeaking Hip Prostheses}

Ehsan Askari ${ }^{1}$

Australian School of Advanced Medicine,

Macquarie University,

Sydney, NSW 2109, Australia;

Department of Mechanical Engineering,

School of Engineering,

University of Minho,

Braga P-4704-553, Portugal

e-mail: ehsanaskary@gmail.com

\section{Paulo Flores}

Department of Mechanical Engineering,

School of Engineering,

University of Minho,

Braga P-4704-553, Portugal

\section{Danè Dabirrahmani}

Australian School of Advanced Medicine,

Macquarie University,

Sydney NSW 2109, Australia

\section{Richard Appleyard}

Australian School of Advanced Medicine,

Macquarie University,

Sydney NSW 2109, Australia

A ceramic-on-ceramic $(\mathrm{CoC})$ hip prosthesis with clearance is modeled as a multibody dynamics system for the purpose of studying hip squeaking. A continuous contact force model provides the intrajoint forces developed at the hip joint. Friction effects due to the relative motion are also considered. A FFT analysis of the audible sounds from CoC hip acceleration is carried out to analyze hip squeaking. The effects of friction, hip implant size, and the head initial position on hip squeaking and the trajectory of femoral head are analyzed and discussed. It was shown that the causes of hip squeaking are stick/slip, friction-induced vibration, and the femoral head angular speed and force changes.

[DOI: $10.1115 / 1.4028109$ ]

Keywords: hip squeaking, multibody dynamics

\section{Introduction}

Trauma, rheumatoid arthritis, and ostheoarthritis can severely impair hip joint function resulting in pain and constrained motion. Often affected hip joints are replaced by biomaterials, which are assumed to be one of the best clinical treatment options. Alumina ceramic bearings as a great option to be used for total hip arthoplasty are bioinert and have high hardness, perfect chemical inertia and low coefficient of friction [1]. From the reliability point of view, it has been suggested that surgeons, faced especially with young and active patients, should consider ceramics, as a safe hard-on-hard bearing surface suitable for implantation [2]. However, the occurrence of audible squeaking in some patients with $\mathrm{CoC}$ hip implants is a cause for concern. Hip squeaking has been

${ }^{1}$ Corresponding author.

Contributed by the Design Engineering Division of ASME for publication in the Journal of Computational and Nonlinear Dynamics. Manuscript received November 9, 2013; final manuscript received July 27, 2014; published online January 12, 2015. Assoc. Editor: Tae-Won Park. reported with a prevalence rate of $1 \%$ to $20 \%$ [1]. The hip squeaking has been associated with high friction $[3,4]$. Moreover, stickslip phenomenon between the head and cup has been reported as a possible cause of squeaking $\mathrm{CoC}$ bearings without lubrication [5]. Sanders et al. [6] reproduced hip squeaking subjected to lubrication condition in vitro. Suboptimal lubrication caused adverse tribological condition, which was a likely cause of squeaking in hip arthroplasty [7].

A femoral stem and head (i.e., the femoral components) and a cup and its lining (i.e., the acetabular components) constitute the mechanism of hip prostheses. This is a biojoint with a clearance defined as a difference between the cup and head radii. The existence of clearance in joints causes dynamic impact loading, affecting the system transfer capacity. The initial conditions of the head versus the cup affect the system trajectory significantly due to the nonlinear nature of the system. It must be highlighted that previous investigations have not studied dynamics and vibration of artificial hip joints, taking the influences of contact/impact mechanics, friction-induced vibration, and the femoral head mass into account. Furthermore, the effect of the femoral head's initial condition on hip prosthesis trajectory and hip squeaking has not been investigated.

Thus, to address the issues mentioned above, a dynamic multibody model of hip prostheses with clearance is developed in the present work. The method utilizes the cross section idea recently introduced by Askari et al. [8] to consider the influence of friction-induced vibration on contact pressure and moment of hip implants. ISO14242-1 testing standard profiles of the femoral head forces and angular rotation over a normal walking cycle are also utilized. The femoral head trajectory, stick-slip phenomenon and hip squeaking can be predicted. The friction-induced vibration and contact-impact events are taken into consideration as external generalized forces. An explicit numerical approach is used to solve this nonlinear dynamic problem. A FFT frequency analysis of the audible sounds from $\mathrm{CoC}$ hip acceleration is also carried out to assess squeaking frequencies. Finally, the effects of hip implant size and clearance, initial condition and friction on the system dynamic are analyzed.

\section{Multibody Dynamic Approach}

In order to determine the governing equations of the femoral head motion, the cross section proposed by Askari et al. [8] is used, which results in considering a typical head/cup joint shown in Fig. $1 . O_{\mathrm{j}}$ indicates the head center, $O_{\mathrm{b}}$ the cup center, and the cup is assumed to be stationary. $P_{\mathrm{j}}$ and $O_{\mathrm{b}}$ denote the contact points on the head and cup, respectively. $r$ is the magnitude of clearance vector and $\alpha$ is its orientation, which are functions of

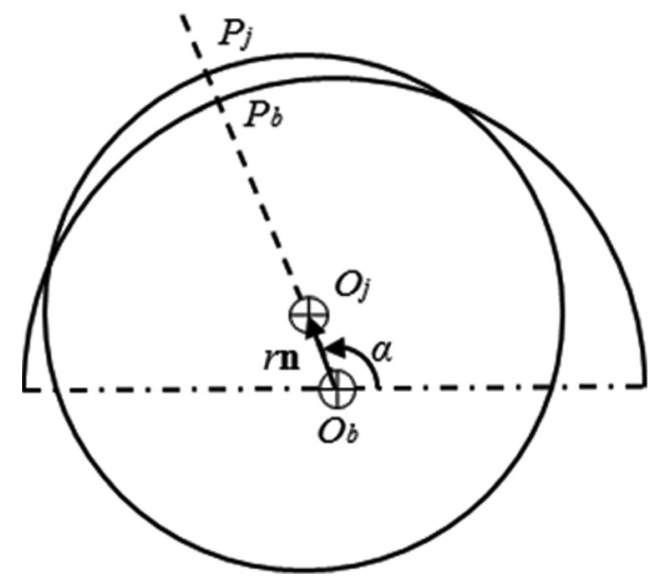

Fig. 1 A schematic representation of the head and liner in contact 


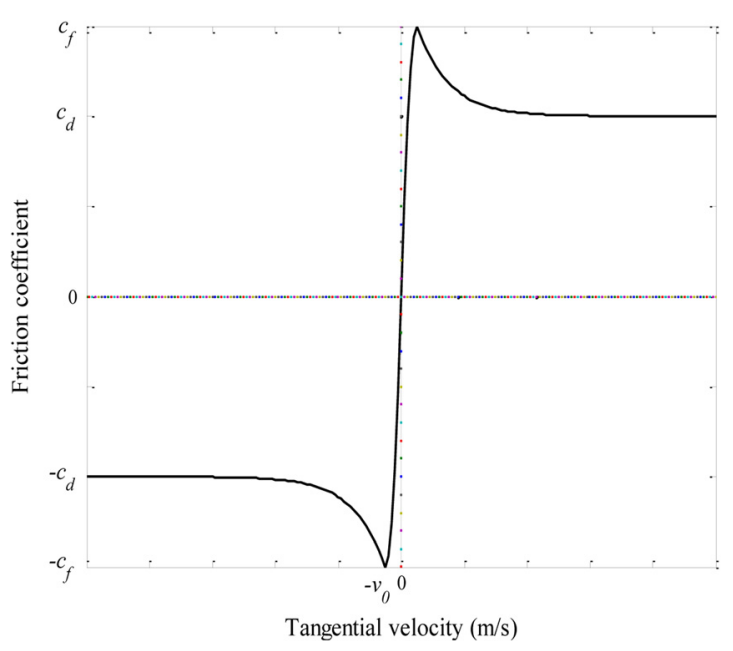

Fig. 2 Stribeck characteristic for dry friction [8]

generalized coordinates selected to describe the configuration of multibody system.

Unit normal and tangential vectors to the collision plane can be expressed as

$$
\begin{gathered}
\mathbf{n}=\cos \alpha \mathbf{i}+\sin \alpha \mathbf{j} \\
\mathbf{t}=-\sin \alpha \mathbf{i}+\cos \alpha \mathbf{j}
\end{gathered}
$$

The evaluation of normal contact and tangential friction forces requires the evaluation of relative tangential and normal velocities of contact points. Position vectors of potential contact points can be written as follows:

$$
\begin{gathered}
\mathbf{r}_{P_{\mathrm{j}}}=\mathbf{r}_{O_{\mathrm{j}}}+\mathbf{r}_{P_{\mathrm{j}} / O_{\mathrm{j}}} \\
\mathbf{r}_{P_{\mathrm{b}}}=\mathbf{r}_{P_{\mathrm{b}} / O_{\mathrm{b}}}
\end{gathered}
$$

in which $\mathbf{r}_{P_{\mathrm{j}}}$ and $\mathbf{r}_{P_{\mathrm{b}}}$ are the position vectors of contact points on the head and cup with respect to the global reference frame placed
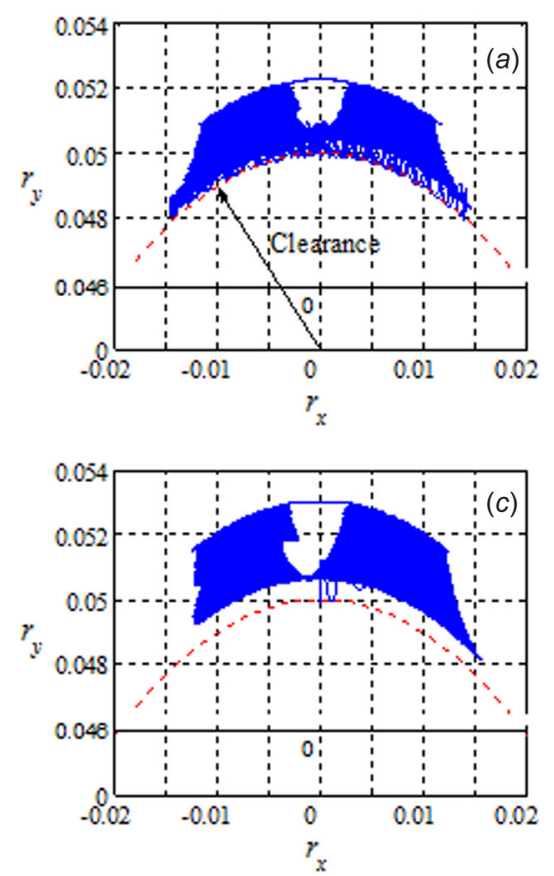

at the cup center, $O_{\mathrm{b}}$. The distance vector between the contact points of the cup and head is given by

$$
\mathbf{r}_{P_{\mathrm{j}} / P_{\mathrm{b}}}=\mathbf{r}_{O_{\mathrm{j}}}+\mathbf{r}_{P_{\mathrm{j}} / O_{\mathrm{j}}}-\mathbf{r}_{P_{\mathrm{b}} / O_{\mathrm{b}}}
$$

where

$$
\mathbf{r}_{O_{\mathrm{j}}}=r \mathbf{n}
$$

Differentiating Eq. (5) with respect to time yields

$$
\mathbf{v}_{P_{\mathrm{j}} / P_{\mathrm{b}}}=\frac{\mathrm{d}}{\mathrm{d} t}\left(\mathbf{r}_{P_{\mathrm{j}} / P_{\mathrm{b}}}\right)=\frac{\mathrm{d}}{\mathrm{d} t}(r \mathbf{n})+\boldsymbol{\Omega}_{\mathrm{j}} \times \mathbf{r}_{P_{\mathrm{j}} / O_{\mathrm{j}}}-\boldsymbol{\Omega}_{\mathrm{b}} \times \mathbf{r}_{P_{\mathrm{b}} / O_{\mathrm{b}}}
$$

where $\boldsymbol{\Omega}_{\mathbf{j}}=\omega_{\mathrm{j}} \mathbf{k}$ and $\boldsymbol{\Omega}_{\mathrm{b}}$ is zero because the cup is assumed to be stationary. Additionally, $\mathbf{k}$ is a unit vector such that $\mathbf{n} \times \mathbf{t}=\mathbf{k}$. Thus, the relative velocity of contact points is given by

$$
\mathbf{v}_{P_{\mathrm{j}} / P_{\mathrm{b}}}=\dot{r} \mathbf{n}+\left(r \dot{\alpha}+R_{\mathrm{j}} \omega_{\mathrm{j}}\right) \mathbf{t}=v_{\mathrm{n}} \mathbf{n}+v_{\mathrm{t}} \mathbf{t}
$$

in which $R_{\mathrm{j}}$ is the head radius. The relative penetration depth is determined by

$$
\delta=r-\left(R_{\mathrm{b}}-R_{\mathrm{j}}\right)
$$

where $\left(R_{\mathrm{b}}-R_{\mathrm{j}}\right)$ is defined as joint radial clearance.

The elastic-damping contact force model proposed by Lankarani and Nikravesh [9] is used to treat contact-impact, which is expressed as

$$
\mathbf{F}_{p_{\mathrm{j}}}^{n}=-K \delta^{\frac{3}{2}}\left(1+\frac{3\left(1-c_{\mathrm{e}}^{2}\right)}{4} \frac{\dot{\delta}}{\dot{\delta}^{(-)}}\right) \mathbf{n}
$$

where $\dot{\delta}$ and $\dot{\delta}^{(-)}$are the relative penetration velocity and initial impact velocity, respectively, and $c_{\mathrm{e}}$ is the restitution coefficient. The generalized stiffness parameter $K$ depends on the geometry and physical properties of the contacting surfaces [9].

Moreover, the tangential contact forces can be evaluated as friction force from modified Coulomb friction law
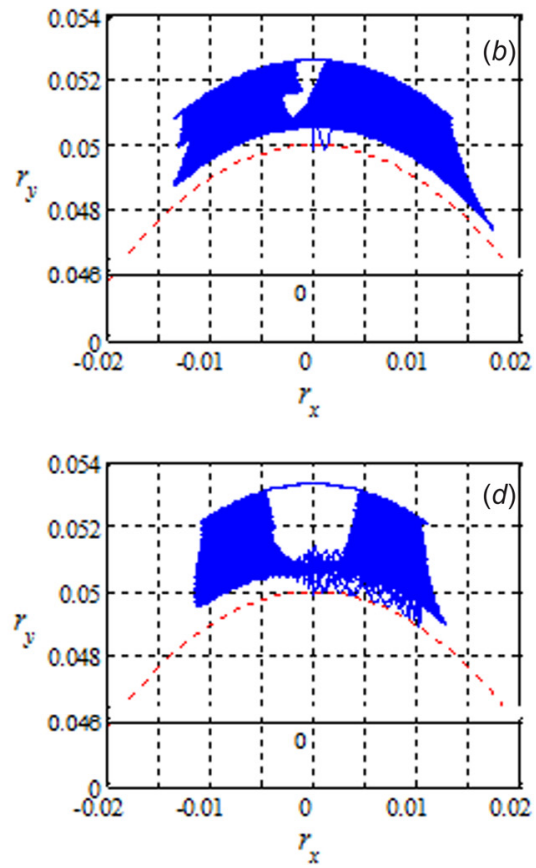

Fig. 3 The head trajectory for initial condition of $(0,0.0499) \mathrm{mm}$ and different cup radii: (a) $25,(b) 20,(c) 16$, and (d) $14 \mathrm{~mm}$ 

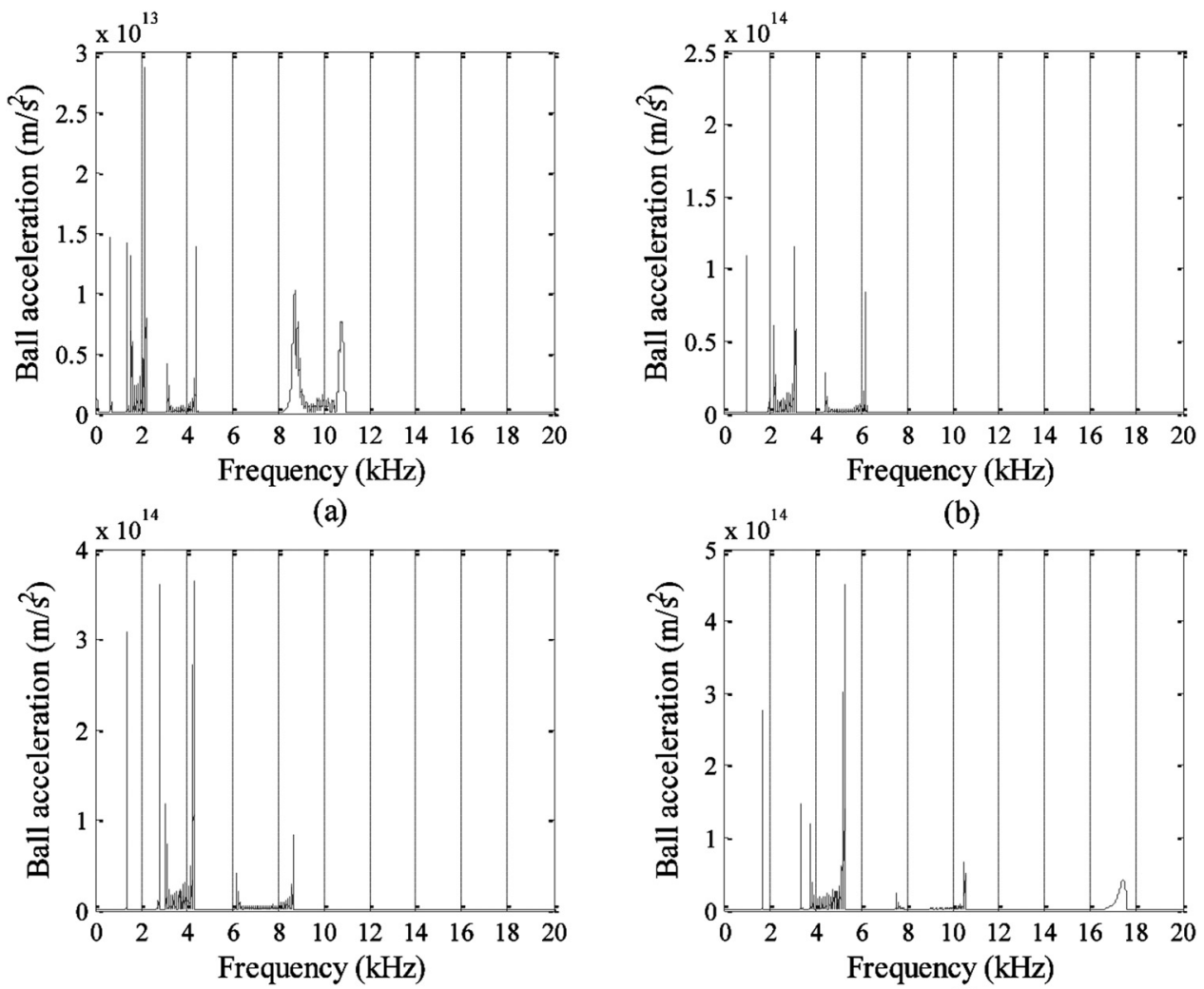

(c)

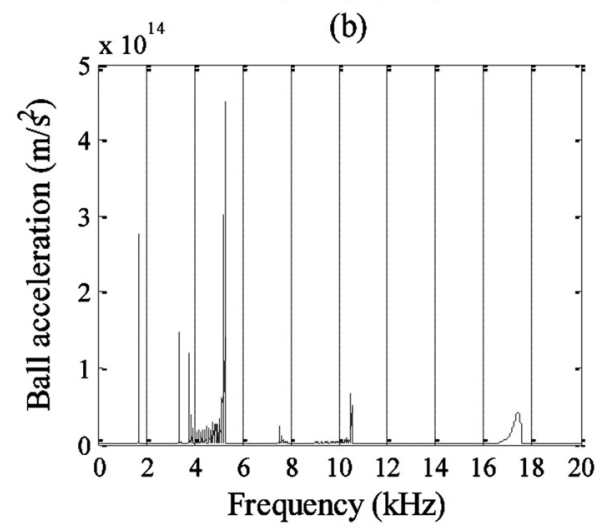

(d)

Fig. 4 FFT analysis with the head initial condition of $(0,0.0499) \mathrm{mm}$ for different cup radii: (a) 25, (640, 1360-1600, 2250, 3200 Hz), (b) 20, (1000, 2000-2200,3100,4420 Hz), (c) 16, (1400, $2800-3100,4330,6200 \mathrm{~Hz})$, and (d) $14 \mathrm{~mm},(1700,3400-3800,5250,7300 \mathrm{~Hz})$
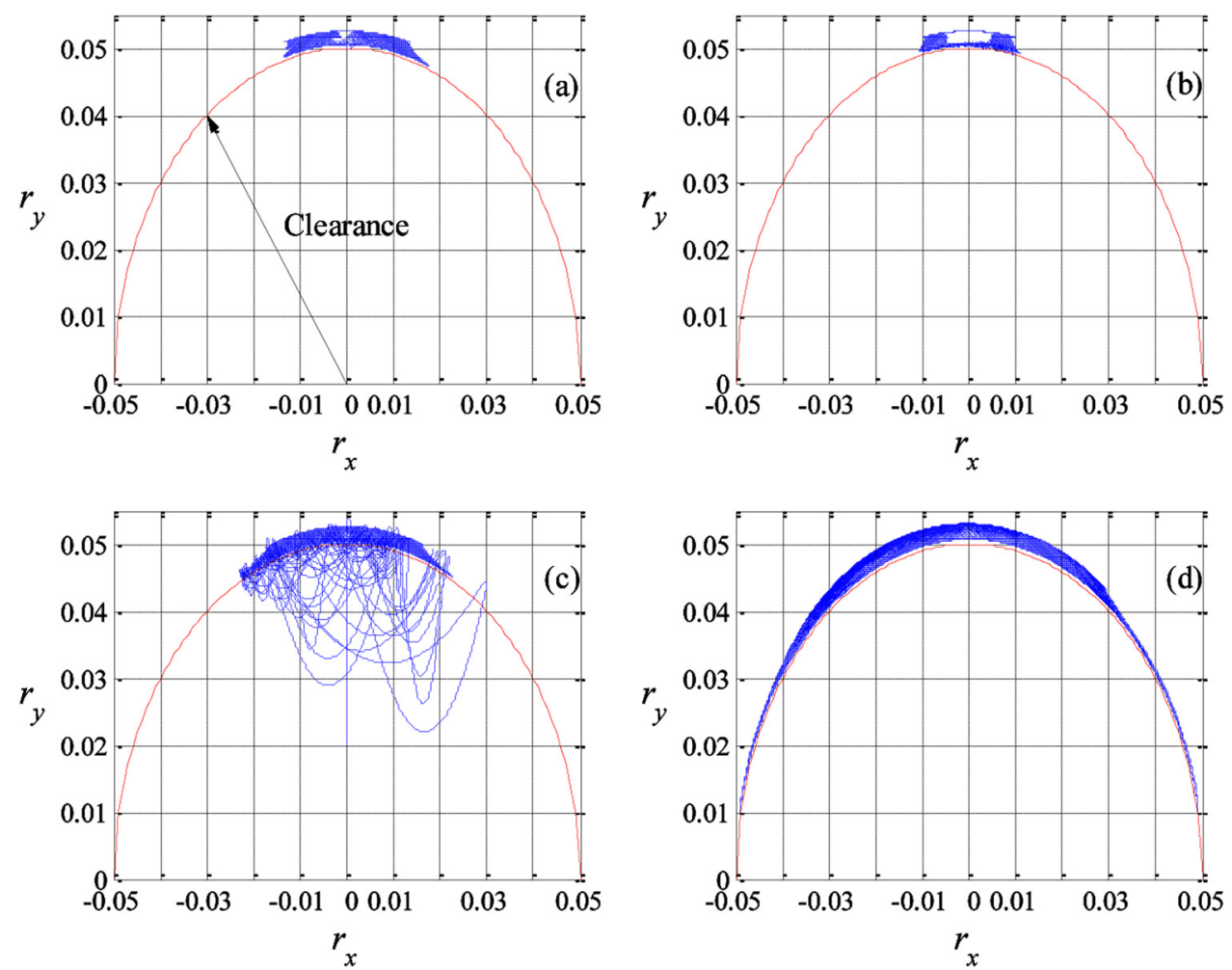

Fig. 5 The head trajectory for different initial conditions: $(a)(0,0.0499),(b)(0.01,0.0489),(c)(0$, $0.02)$, and (d) $(0.0489,0.01) \mathrm{mm}$ 

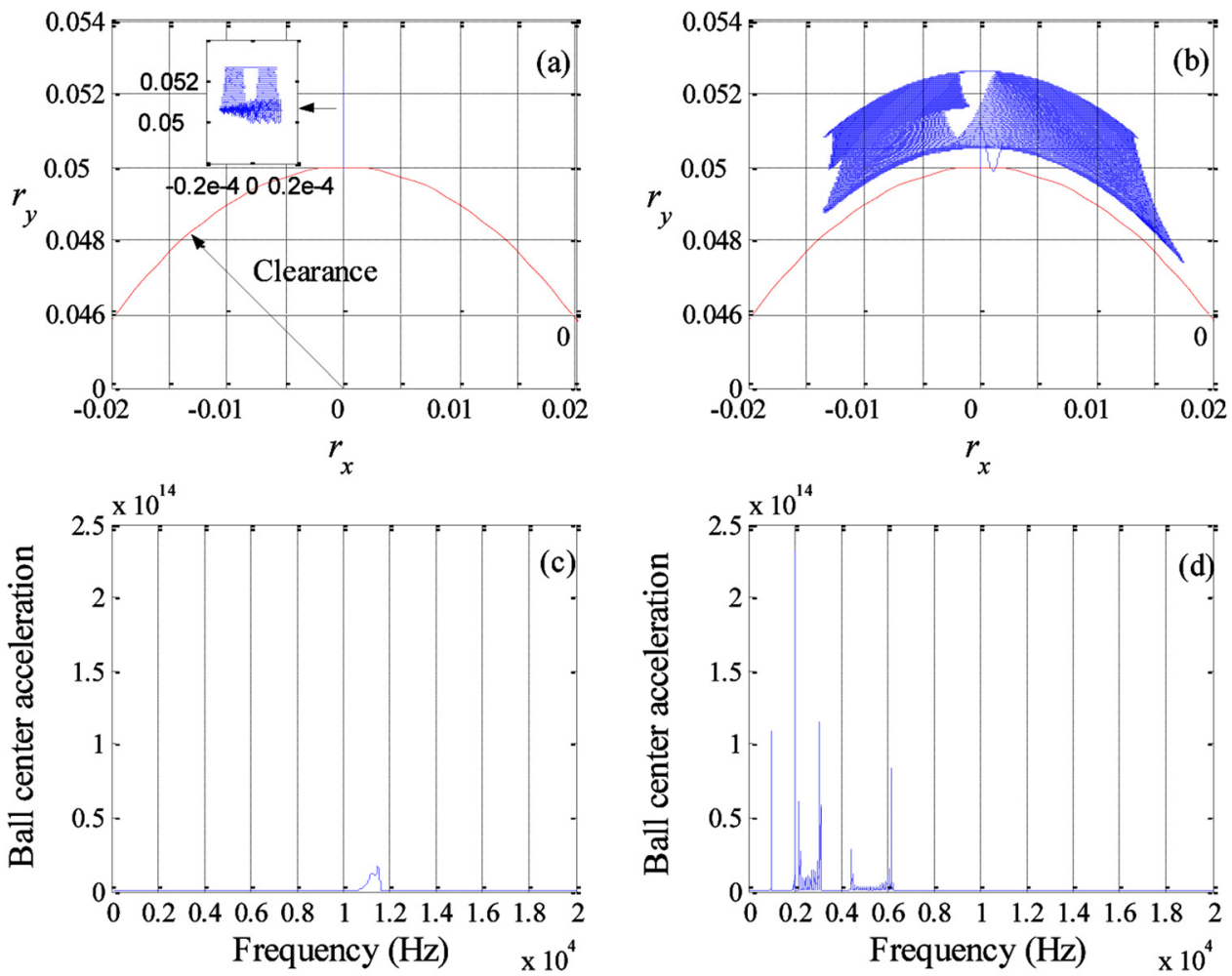

Fig. 6 The head trajectory with the initial condition of $(0,0.0499) \mathrm{mm}$ : (a) II and (b) I. FFT analysis of hip implants $(c)$ II and $(d) I\left(I: C_{f} / C_{d}=0.15 / 0.1\right.$, II: $\left.C_{f} / C_{d}=0.00015 / 0.0001\right)$.

$$
\mathbf{F}_{p_{\mathrm{j}}}^{t}=-\mu\left(v_{\mathrm{t}}\right)\left\|\mathbf{F}_{p_{\mathrm{j}}}^{n}\right\| \mathbf{t}
$$

where $v_{\mathrm{t}}$ represents the tangential velocity and $\mu\left(v_{\mathrm{t}}\right)$ denotes the friction coefficient, which is defined as the Stribeck model and shown in Fig. 2.

By transferring normal contact and friction forces to the femoral head center, the equations of motion are derived using Newton's Second law, which can be written as [8]

$$
\left[\begin{array}{cc}
m & 0 \\
0 & m
\end{array}\right]\left[\begin{array}{c}
\ddot{x} \\
\ddot{y}
\end{array}\right]=\left[\begin{array}{c}
f_{x}+\left(\mathbf{F}_{p_{\mathrm{j}}}^{\mathrm{t}}+\mathbf{F}_{p_{\mathrm{j}}}^{\mathrm{n}}\right) \cdot \mathbf{i}\langle\delta\rangle^{0} \\
f_{y}+\left(\mathbf{F}_{p_{\mathrm{j}}}^{\mathrm{t}}+\mathbf{F}_{p_{\mathrm{j}}}^{\mathrm{n}}\right) \cdot \mathbf{j}\langle\delta\rangle^{0}-\mathrm{mg}
\end{array}\right]
$$

The resulting equations are nonlinear and must be solved by using a numerical method.

\section{Results and Discussion}

In the present study, a multibody dynamic model of a $\mathrm{CoC}$ artificial hip joint is considered. The geometrical and material properties of the femoral head and acetabular cup are the same as Ref. [8]. The main vertical load and flexion/extension angular velocity were extracted from ISO14242-1 testing standard profiles. The dynamic respond of the system is obtained by solving the equations of motion described above employing the adaptive Runge-Kutta-Fehlberg method [8].

3.1 Effects of Hip Implant Size. The head trajectory and squeaking are illustrated over the gait cycle for four different cup and head radii, as it is shown in Figs. 3 and 4, respectively. The squeaking frequencies are also reported in the caption of Fig. 4. As observed, $\mathrm{CoC}$ fundamental squeaking frequencies are in the range of $400-7500 \mathrm{~Hz}$, being consistent with clinical data [10]. A decrease in the head size increases squeaking frequencies since the femoral head mass decreases. Additionally, the penetration depth increases as hip size decreases due to the reduction of contact area and, therefore, the increase of contact pressure, conforming to the finite element analysis by Meng et al. [11] Head trajectories are similar for different hip implant sizes generally, see Fig. 3 .

3.2 Effects of Initial Position. The femoral head initial position can change due to either the head and cup separation or the disruption of fluid-film lubrication, which consequently results in the impact between the femoral head and cup. Figure 5 shows the head trajectory for different initial conditions during a normal walking cycle. The head impacts and rebounds from the cup surface several times before sitting in the cup surface, as illustrated in Fig. 5(c). Figures 5(a) and 5(b) depict the head moves across a smaller area of the cup surface when compared to the plot in Fig. $5(d)$. This phenomenon can affect the wear process on bearing

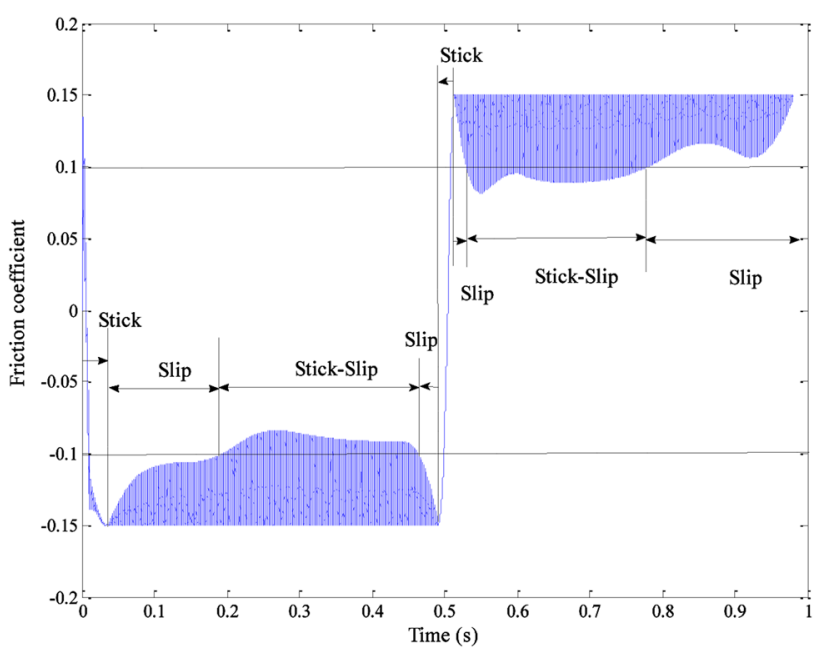

Fig. 7 Stick/slip phase intervals over the gait cycle 


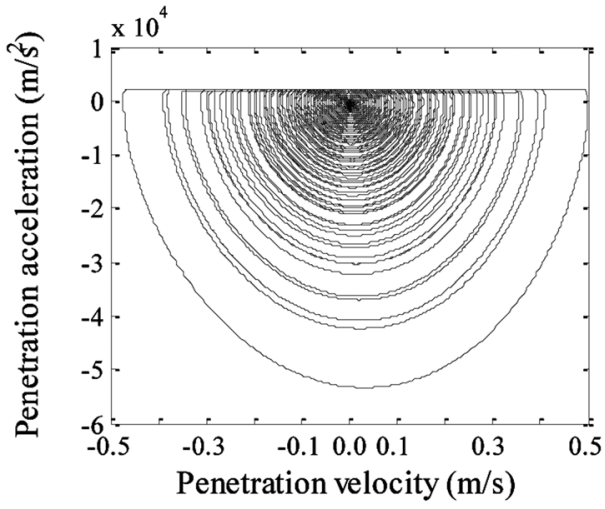

(a)

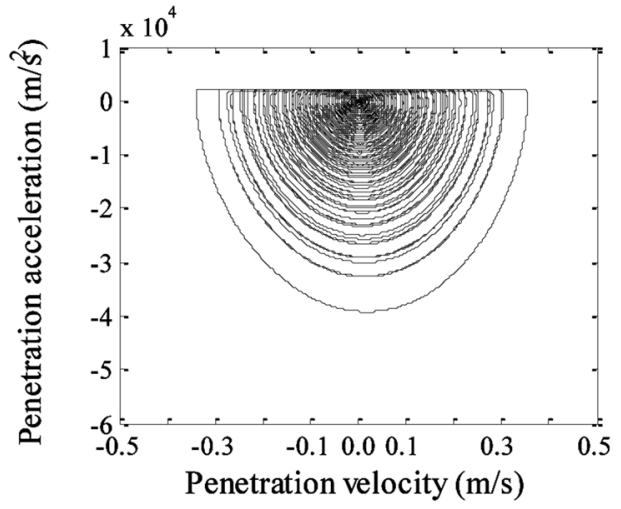

(b)

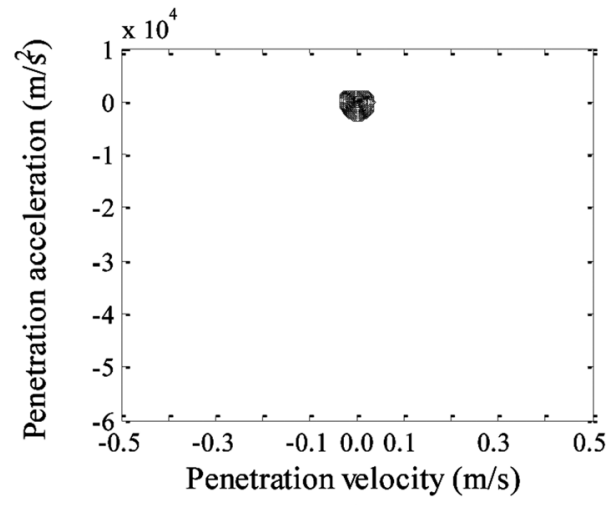

(c)

Fig. 8 Phase portrait diagrams for different clearance sizes: (a) 0.1, (b) 0.05 , and (c) $0.02 \mathrm{~mm}$
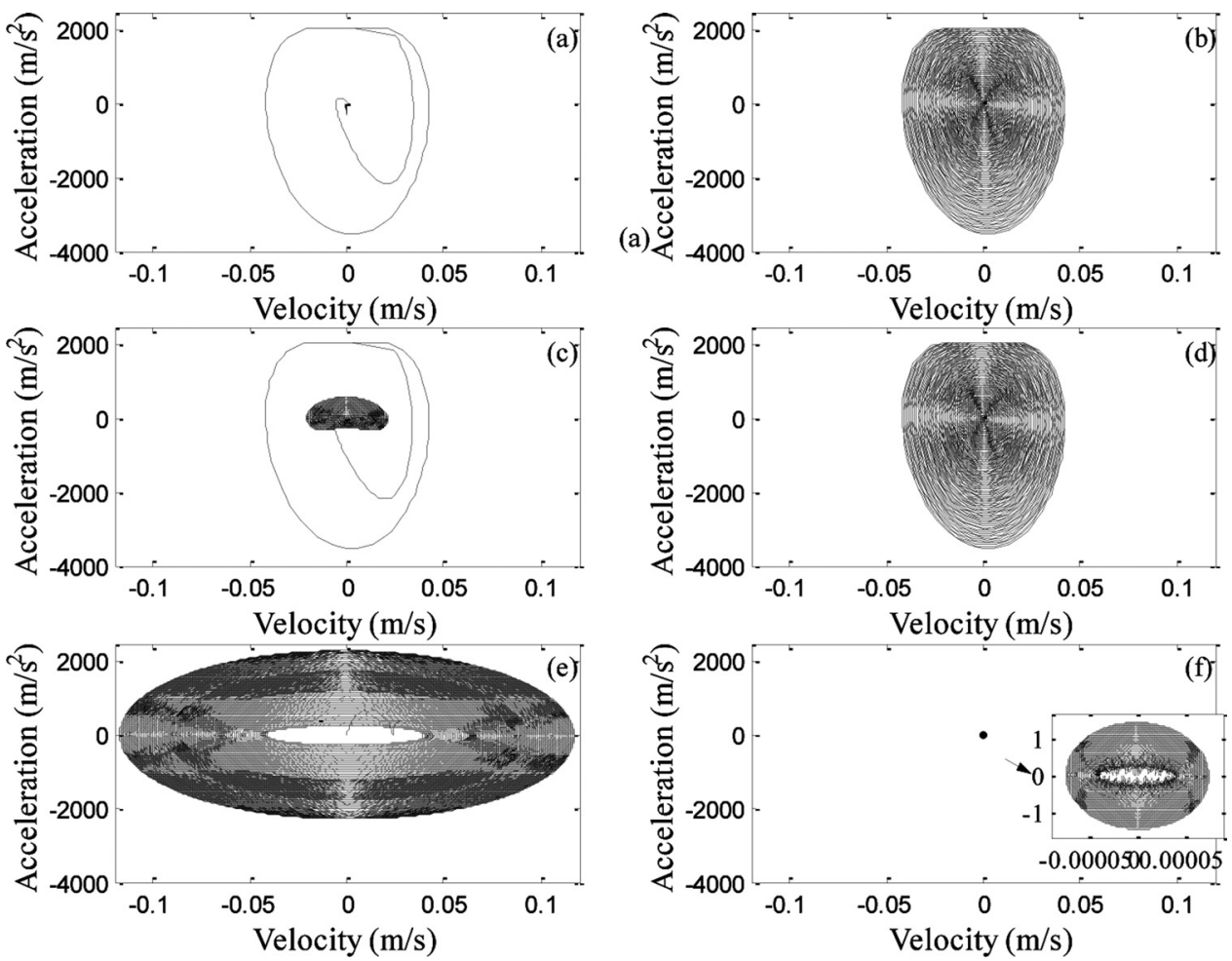

Fig. 9 Penetration acceleration versus velocity: $(a)$ I and $(b)$ II. Vertical acceleration versus velocity, $(c)$ I and $(d)$ II. Horizontal acceleration versus velocity $(e) I$ and $(f)$ II $\left(\mathrm{I}: C_{f} / C_{\mathrm{d}}=0.15 / 0.1\right.$, II: $\left.C_{\mathrm{f}} / C_{\mathrm{d}}=0.00015 / 0.0001\right)$. 


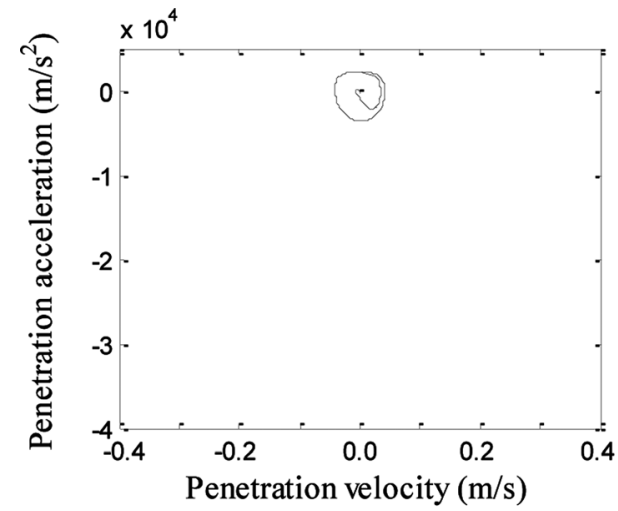

(a)

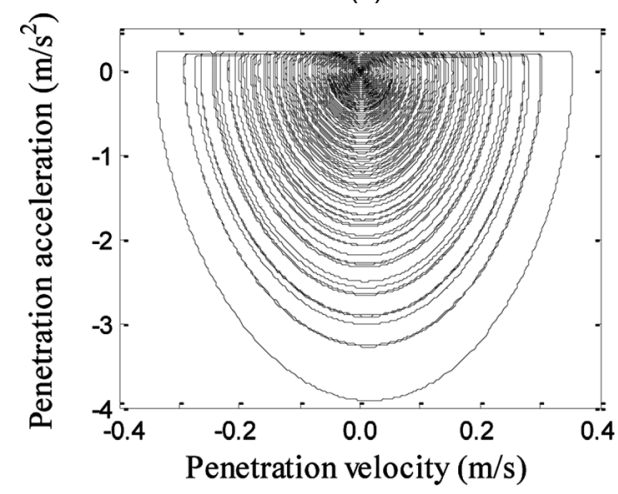

(c)

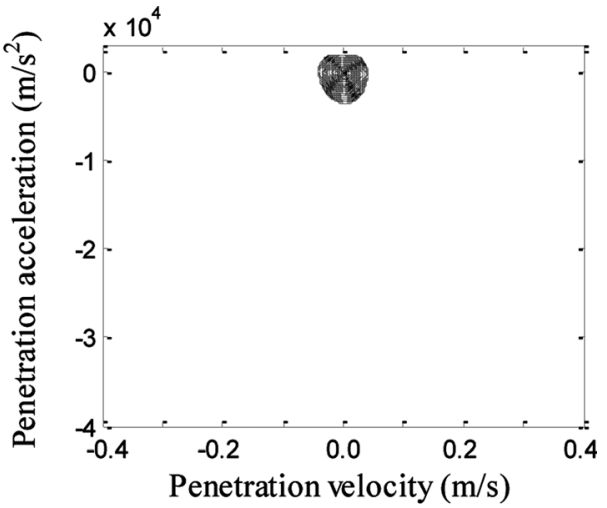

(b)

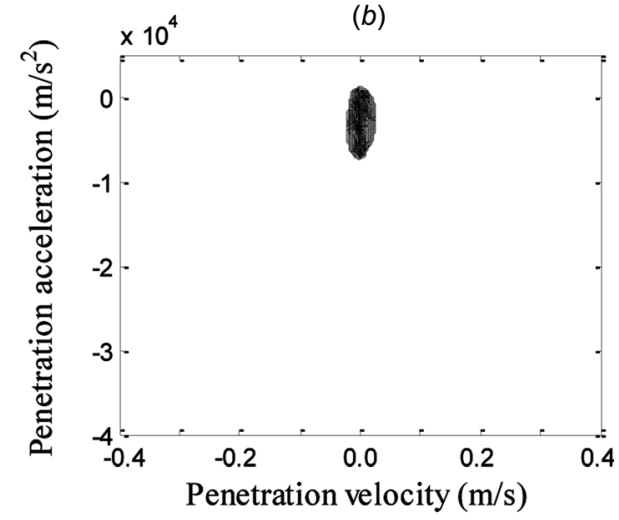

(d)

Fig. 10 Phase portrait diagrams for different initial conditions: $(a)(0,0.0499),(b)(0.01,0.0489)$, (c) $(0,0.02)$, and $(d)(0.0489,0.01) \mathrm{mm}$

surfaces. Moreover, initial conditions do not have significant influences on squeaking frequencies and penetration depths.

3.3 Influence of Friction. By observing Fig. 6, it can be concluded that the main cause of hip squeaking is high friction, which corroborates the literature $[4,5]$. In other words, friction results in friction-induced vibration and stick/slip phenomenon, which leads to hip squeaking. When friction increases a greater part of the cup surface undergoes contact with the head surface, see Figs. 6(a) and $6(b)$, which affects wear process on bearing surfaces. Figure 7 shows stick/slip phase intervals of the femoral head motion during the gait cycle. The head can roll in both stick/slip phases. The friction coefficient has an oscillating behavior due to the relative velocity between the femoral head and cup, stick/slip and negative gradient seen in the Stribeck friction curve.

3.4 Phase Portrait Diagrams. Phase portrait diagrams are utilized to study the type of dynamic response observed in the hip model for different scenarios. Figure 8 depicts the influence of clearance size on the dynamic response type of hip implant. Figures $8(a)$ and $8(b)$ show chaotic behaviors of head motion, which are extremely sensitive to initial conditions and densely filled by orbits or points in the phase portrait diagram [12]. This chaotic response suggests that head/cup impacts followed by rebounds occur. A quasi-periodic behavior can be observed in Fig. 8(c) since quasi-periodic orbits fill up phase portrait diagram in a fully predictable manner. This behavior is due to the fact that quasi-periodic behavior does not depend on initial conditions sensitively. Generally, when clearance size decreases, dynamic behavior tends to be smoother, in the measure that the system response tends to be closer to the ideal case meaning that the femoral head and cup experience continuous or permanent contact.

The presence of friction reduces the peak force values associated with head/cup impact. For a low friction coefficient, the system response is chaotic as demonstrated by phase portrait diagram trajectory spread, see Fig. 9(b). Figure $9(a)$ shows a quasiperiodic motion with orbits filling up the phase portrait diagram in a predictable manner. Moreover, it can be seen from Figs. 9(e) and $9(f)$ that the motion of head in $x$-direction is limited as friction decreases. Figure 10 indicates the effect of head initial position on its trajectory. The ball trajectory is chaotic when the head motion starts at $(0,0.02) \mathrm{mm}$. However, as illustrated in Figs. $10(b)$ and $10(d)$, the motion is quasi-periodic, and the ball motion is more periodic in Fig. 10(a). It can be stated that hip dynamic response is nonlinear due to the friction effect, intermittent motion, contact/ impact mechanics, and high locomotion range. Under certain conditions, the system exhibits a chaotic response. In conclusion, it can be found that the dynamics of artificial hip joint is sensitive to clearance size, friction coefficient, and initial position of the head. Even with a small change in one of these parameters the response of the system can shift from chaotic to quasi-periodic or periodic and visa versa.

\section{Conclusions}

A planar multibody dynamic approach to model artificial hip joints with clearance has been presented in this work. The effect of friction-induced vibration, stick/slip, and contact/impact mechanics on the system dynamic was captured. The method enables user to model the hip implant motion with either standard hip force and angular motion or in vivo ones. Utilizing the proposed approach, hip squeaking of $\mathrm{CoC}$ hip implants can successfully be analyzed. Results obtained from the developed method were consistent with in vivo outcomes. It was concluded that the femoral head vibrates due to the head angular speed and force changes, stick/slip and friction-induced vibration, which is a result of high friction. The decrease of hip implant size increased both hip squeaking frequencies and penetration depth. Initial conditions did not have significant influence on squeaking frequencies. Stick and slip phase intervals of the femoral head movement were also 
observed. With a small change in one of these parameters, the response of the system can shift from chaotic to quasi-periodic or periodic and visa versa.

\section{References}

[1] Restrepo, C., Parvizi, J., Kurtz, S. M., Sharkey, P. F., Hozack, W. J., and Rothman, R. H., 2008, "The Noisy Ceramic Hip: Is Component Malpositioning the Cause?," J. Arthroplasty, 23(5), pp. 643-649.

[2] Capello, W. N., D'Antonio, J. A., Feinberg, J. R., Manley, M. T., and Naughton, M., 2008, "Ceramic-on-Ceramic Total Hip Arthroplasty," J. Arthroplasty, 23(7), pp. 39-43.

[3] Weiss, C., Hothan, A., Huber, G., Morlock, M. M., and Hoffmann, N. P., 2012, "Friction-Induced Whirl Vibration: Root Cause of Squeaking in Total Hip Arthroplasty," J. Biomech., 45(2), pp. 297-303.

[4] Fan, N., and Chen, G. X., 2012, "Numerical Study of Squeaking Suppresses for Ceramic-on-Ceramic Hip Endoprosthesis," Tribol. Int., 48, pp. 172-181.

[5] Currier, J. H., Anderson, D. E., and Van Citters, D. W., 2010, "A Proposed Mechanism for Squeaking of Ceramic-on-Ceramic Hips," Wear, 269(11-12), pp. 782-789.
[6] Sanders, A., Tibbitts, I., and Brannon, R., 2012, "Concomitant Evolution of Wear and Squeaking in Dual-Severity, Lubricated Wear Testing of Ceramic-on-Ceramic Hip Prostheses," J. Orthop. Res., 30(9), pp. 1377-1383.

[7] Brockett, C. L., Williams, S., Jin, Z., Isaac, G. H., and Fisher, J., 2013, "Squeaking Hip Arthroplasties: A Tribological Phenomenon," J. Arthroplasty, 28(1), pp. 90-97.

[8] Askari, E., Flores, P., Dabirrahmani, D., and Appleyard, R., 2014, "Study of the Friction-Induced Vibration and Contact Mechanics of Artificial Hip Joints," Tribol. Int., 70, pp. 1-10.

[9] Lankarani, H. M., and Nikravesh, P. E., 1990, "A Contact Force Model With Hysteresis Damping for Impact Analysis of Multibody Systems," ASME J. Mech. Des., 112(3), pp. 369-376.

[10] Walter, W. L., Waters, T. S., Gillies, M., Donohoo, S., Kurtz, S. M., Ranawat, A. S., Hozack, W. J., and Tuke, M. A., 2008, "Squeaking Hips," J. Bone Jt. Surg. Am., 90(4), pp. 102-111.

[11] Meng, Q., Liu, F., Fisher, J., and Jin, Z., 2013, "Contact Mechanics and Lubrication Analyses of Ceramic-on-Metal Total Hip Replacements," Tribol. Int., 63, pp. 51-60.

[12] Wiggins, S., 1990, Introduction to Applied Nonlinear Dynamical Systems and Chaos, Springer-Verlag, New York. 